\section{PAS positive pseudofungi: a diagnostic pitfall}

Sir,

Accurate diagnosis of pseudofungi in histology specimens is imperative for avoiding unnecessary treatment. Special stains are key to separating true fungi from their mimics. Periodic acid-Schiff (PAS) stain is a common stain used in the diagnosis of fungi, typically highlighting fungal cell walls, but also staining other polysaccharides and mucins. We report a case of striking and extensive pseudofungi incidentally detected in perigastric lymph nodes.

A 68-year-old woman presented for routine imaging surveillance of a high risk gastric epithelioid gastrointestinal

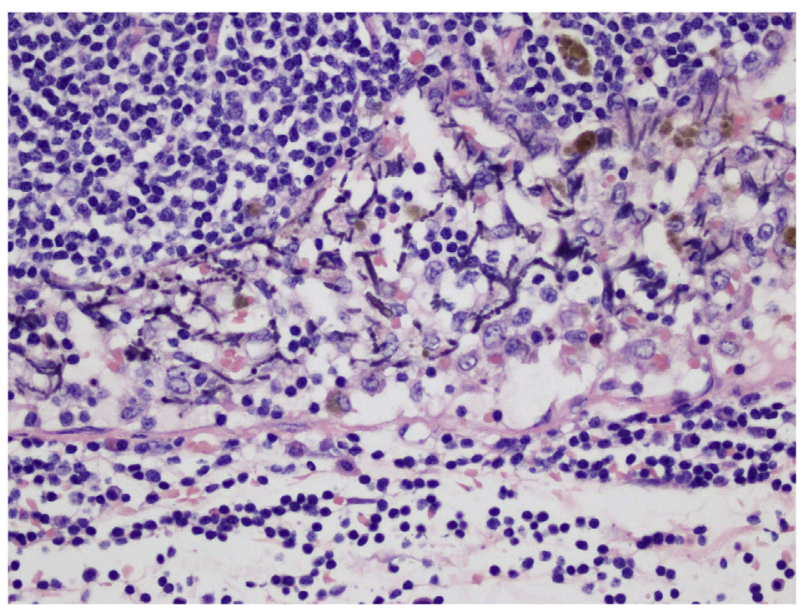

Fig. 1 Pseudofungi presenting as septate, acute to right angled branching structures seen within a lymph node subcapsular sinus (H\&E). stromal tumour, resected 5 years prior and treated with adjuvant imatinib. Computed tomography (CT) and positron emission tomography (PET) imaging showed evidence of recurrent disease adjacent to the gastric anastomosis, with no evidence of nodal disease. She was systemically well and underwent a subtotal gastrectomy with intraoperative findings of an exophytic lesion at the greater curve of the distal stomach, with adhesions to the anterior abdominal wall and liver.

Grossly, the specimen comprised a $130 \mathrm{~mm}$, multilobed, fleshy and haemorrhagic tumour arising from the serosal surface of the stomach. Three lymph nodes were present in the perigastric fat and were grossly unremarkable, less than 6 $\mathrm{mm}$ in maximal dimension. Histological examination of the tumour showed a partially necrotic epithelioid gastrointestinal stromal tumour, which was strongly positive for DOG-1 immunohistochemistry.

Examination of the lymph nodes showed no evidence of metastatic disease. Follicular architecture was preserved. In the subcapsular sinuses, there were abundant hyphae-like structures with mild variation in thickness, demonstrating septa and acute to right angle branching (Fig. 1). There were scattered haemosiderin laden macrophages in the background. An initial panel of stains was performed, with the structures positive for PAS and negative for Grocott's methenamine silver (GMS). Perls' stain was strongly positive in the hyphae-like structures, as well as in the haemosiderin laden macrophages. Supplementary stains showed positivity for Alizarin red, orcein, reticulin, Sirius red, and Van Gieson stains (Fig. 2).

This pattern of staining suggests the pseudofungi consist of collagen fibres with deposition of iron and calcium. Similar structures have been observed within lymph nodes in rare case reports, also typically demonstrating a PAS positive,

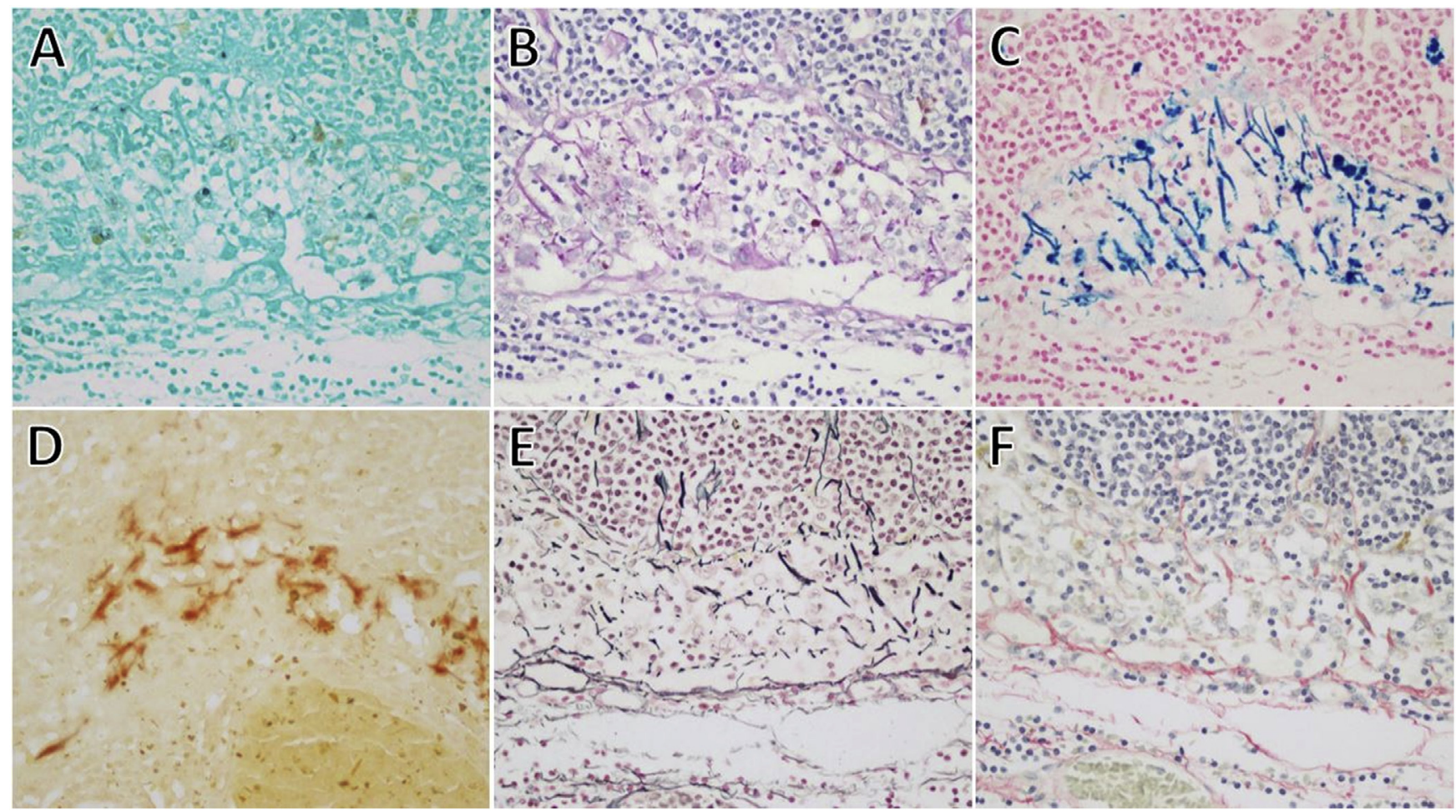

Fig. 2 Special stains used for diagnosis of pseudofungi. (A) Grocott's methenamine silver negative, (B) periodic acid-Schiff positive, (C) Perls' positive, (D) Alizarin red positive, $(\mathrm{E})$ reticulin positive, $(\mathrm{F})$ Sirius red positive. 
Perls' positive, and GMS negative staining pattern. ${ }^{1-4}$ Analysis by energy dispersive radiographic microanalysis of two cases ${ }^{1,2}$ showed the pseudofungi contained phosphorus, sulphur, potassium, calcium, and iron, with ultrastructural examination showing that septation was a result of fracturing of the calcified structures. Interestingly, one of these cases also involved nodes associated with resection of a large haemorrhagic gastrointestinal stromal tumour ${ }^{2}$; other cases have been associated with malignant melanoma ${ }^{1,4}$ and large cell lung carcinoma. ${ }^{3}$ It is theorised that haemorrhage associated with such tumours may lead to haemosiderin deposition in the draining nodes, with eventual degradation leading to iron deposition on collagen or reticulin fibres. ${ }^{2,4}$ This is supported by the presence of background Perls' positive haemosiderin laden macrophages observed in our case.

Awareness of fungal mimics is important to avoid misdiagnosis of a fungal infection. In addition to histological examination, a panel of special stains should be employed to assist to differentiate fungal organisms from pseudofungi. Our case highlights the strong resemblance of pseudofungi to true fungi, and the utility of special stains for accurate diagnosis.

Conflicts of interest and sources of funding: The authors state that there are no conflicts of interest to disclose.

\section{Stephanie Lau, David Williams}

\section{Austin Pathology, Austin Hospital, Heidelberg, Australia}

Contact Dr Stephanie Lau.

E-mail: stephanie.1au@austin.org.au

1. Connelly J, Ro JY, Cartwright Jr J. Pseudofungi in a lymph node. A case report with energy dispersive X-ray elemental analysis. Arch Pathol Lab Med 1991; 11: 1166-8

2. Song DE, Kahn AG, Khang SK, Ro JY. Pseudofungi in pericolic lymph nodes. Arch Pathol Lab Med 2005; 129: e97-100.

3. Teague MD, Tham KT. Hyphalike pseudofungus in a lymph node. Arch Pathol Lab Med 1994; 1: 95-6.

4. Singh C, Manivel C, Pambuccian SE. Pseudofungi: coral shapes and bamboo sticks in lymph node sinuses. Int J Surg Pathol 2010; 18: 68-9.

DOI: https://doi.org/10.1016/j.pathol.2020.08.016

\section{Intranuclear inclusions are a distinguishing morphological feature of renal cell carcinoma with leiomyomatous stroma}

Sir,

Renal cell carcinoma with leiomyomatous stroma (RCCLMS) is an uncommon neoplasm classified as a provisional entity in the 2016 World Health Organization classification of kidney tumours. The relationship of RCCLMS to clear cell renal cell carcinoma and clear cell papillary renal cell carcinoma remains unclear, although increasing evidence supports it as a distinct entity with a unique molecular profile. ${ }^{1}$ In particular, RCCLMS does not appear to harbour alterations in chromosome $3 p$ or the $V H L$ gene as seen in conventional RCC. ${ }^{2}$ A recent study showed recurrent mutations of TSC1/TSC2, MTOR, and/or TCEB1 genes in all 14 tested cases of RCCLMS, ${ }^{3}$ supporting the hypothesis that these tumours represent the sporadic counterpart of histologically similar tumours presenting in patients with tuberous sclerosis. Further elucidation of the morphological features of RCCLMS could assist pathologists to identify this rare tumour.

We retrospectively compiled four cases of RCCLMS; three were reported at our institution and one was an external consultation (Table 1). None of the patients had tuberous sclerosis or end stage kidney disease. The tumours were small and confined to the kidney (pT1a), with no recurrence or metastasis documented in the limited follow-up period $(<2$ years). The morphology and immunohistochemical findings were similar to previously reported cases. ${ }^{1}$ The tumours were circumscribed by an irregular pseudocapsule and showed nodules of clear cells with inconspicuous nucleoli arranged in nests, tubules and occasional papillary structures (Fig. 1). Immunohistochemistry revealed a consistent pattern with positive staining for CK7 and CD10 in the epithelium and smooth muscle actin positivity in the leiomyomatous stroma (Fig. 1). In addition, all four cases showed optically clear to pale eosinophilic inclusions within the nuclei of the lesional clear cells (Fig. 2). The inclusions could be appreciated at $200 \times$ magnification and occupied most of the nucleus. Ultrastructural analysis by electron microscopy showed invaginations of the nuclear membrane containing cytoplasmic organelles (Fig. 2) within tumour cells, consistent with intranuclear cytoplasmic inclusions. Tumour cells with intranuclear inclusions varied in proportion across and within the tumours but were present at least focally in all sections examined. Interestingly, one of the patients (Case 1) had three discrete synchronous tumours each resected separately from the same kidney. The RCCLMS, papillary RCC and clear cell papillary RCC each had distinct morphological and immunohistochemical profiles, consistent with previous reports. ${ }^{1}$ Intranuclear inclusions were present within the RCCLMS only.

A search of the literature did not reveal any prior documentation of intranuclear inclusions in RCCLMS, however such inclusions could be appreciated in multiple micrographs of published case reports of RCCLMS (Fig. 2). ${ }^{4-6}$ Intranuclear inclusions have been reported in RCC, with a higher frequency in papillary and chromophobe carcinoma. ${ }^{7}$ However, these inclusions were described as eosinophilic in colour and were associated with tumours of grade $3 / 4$. In contrast, the inclusions in our series were colourless and present in tumours with inconspicuous nucleoli consistent with WHO/ISUP grade 1/2.

Table 1 Clinicopathological features of four renal cell carcinoma with leiomyomatous stroma cases

\begin{tabular}{lllllll}
\hline Case & Sex & Age & Size $(\mathrm{cm})$ & No. tumours & \multicolumn{1}{c}{ Colour } & Stage \\
\hline 1 & M & 50 & 1.4 & $3^{\text {a }}$ & Tan and grey & pT1a \\
2 & F & 53 & 2.6 & 1 & Tan to yellow and grey & pT1a \\
3 & M & 67 & 2.6 & 1 & Tan and grey & pT1a \\
4 & F & 62 & 3.5 & 1 & Tan to yellow & pT1a \\
\hline
\end{tabular}

a The three tumours were renal cell carcinoma with leiomyomatous stroma, papillary renal cell carcinoma and clear cell papillary renal cell carcinoma, each resected separately. 